\title{
L.O.S.V.D.'s of elliptical galaxies from mergers of disk galaxies
}

\author{
A. C. González-García and M. Balcells \\ Instituto de Astrofísica de Canarias, C/ Vía Lactea s/n, 38200 La Laguna, Tenerife, Spain \\ email: cglez@ll.iac.es
}

\begin{abstract}
Modern kinematic diagnostics from high-S/N spectroscopy provide useful tests on the collisionless merger hypothesis for the formation of elliptical galaxies. Bender, Saglia \& Gerhard (1994) show that the skewness of the line-of-sight velocity distributions (LOSVD) has opposite sign to the rotation velocity and follows a distinct pattern. Previous N-body experiments (Bendo \& Barnes 2000, Naab \& Burkert 2001) disagree as to whether the relation is reproduced by the models.

We present first results from an ongoing effort to explain the skewness properties of the LOSVD's of ellipticals. We use large N-body simulations of disk-disk galaxy mergers $\left(10^{6}\right.$ particles), with mass ratios 1:1 and 3:1. Our 1:1 models fail to reproduce the $\mathrm{H} 3$ vs. $V / \sigma$ relation, in agreement with Naab \& Burkert (2001). 3:1 mergers yield trends similar to the observations for large values of $V / \sigma$, but with overall lower $\mathrm{H} 3$ values in the models than in real galaxies. And we fail to reproduce high $\mathrm{H} 3$ values for low $V / \sigma$. So far, it is unclear whether the disagreement is related to the lack of rotation in the bulge components of our initial model galaxies, or to a more general shortcoming of dissipationless mergers to reproduce the properties of real ellipticals.
\end{abstract}

\section{Introduction}

Bender, Saglia \& Gerhard (1994) measured the line-of-sight velocity distributions (LOSVD) of a number of elliptical galaxies up to $1 R_{\text {eff }}$. Bender et al. fit the LOSVD to a Gaussian plus a combination of Gauss-Hermite polynomials. They find an anti-correlation between the H3 parameter, which gives the amplitude of the 3rd-Hermite polynomial, and $V / \sigma$. H3 describes the asymmetry or skewness of the LOSVD from gaussianity. The correlation appears to be more prominent for disky elliptical galaxies. This has been claimed as a signature of the presence of disks inside disky ellipticals (Bender et al. 1994, Naab \& Burkert 2001).

In the hierarchical merging scenario, elliptical galaxies can be formed by a major merger between two spiral galaxies (Toomre \& Toomre 1972). It has been argued that 1:1 mergers between disk galaxies give as a result a body that resembles a boxy elliptical galaxy, while mergers with mass ratios of the order 3:1 give disky ellipticals (Bendo \& Barnes 2000, Naab \& Burkert 2001, Gonzalez-Garcia \& Balcells 2004).

It is therefore quite straightforward to ask whether the correlation found by Bender et al. (1994) can be found by means of mergers of disks. Naab \& Burkert (2001) study this issue by collisionless simulations of mergers of disks. They use galaxy models with mass ratios $1: 1$ and $3: 1$ with a disk:bulge ratio $3: 1$. They find that for $1: 1$ mergers their systems end up being boxy ellipticals not showing the correlation. Neither the 3:1 mergers, although they show disky deviations, do reproduce Bender's correlation. They argue that this is a proof of the dissipational origin of these structures. 

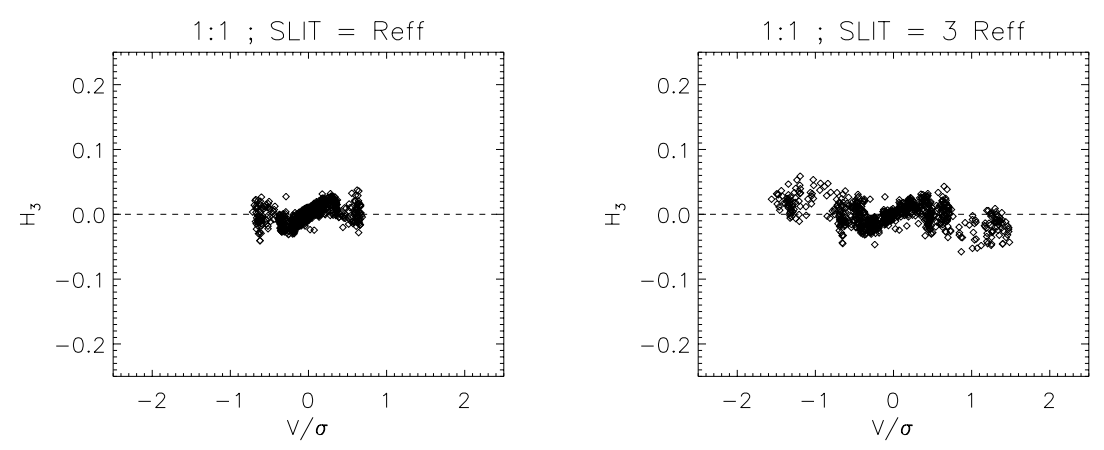

Figure 1. H3 vs. $V / \sigma$ for the 1:1 merger. Two frames are given: the left one was done with a slit with a length equal to the system effective radius. The second is for a slit with $3 R_{e f f}$.

\section{Models}

We build the initial models following Kuijken \& Dubinski (1995). Our initial models have a bulge, a disk and a halo. We use non-dimensional units in our calculations, with $G=1$. The masses of the different components in our initial model are in the ratios: 19:2:1 (halo:disk:bulge). As we can see, the initial mass ratio between disk and bulge is 2:1. Note that our models have a somewhat larger bulge than Naab \& Burkert (2001).

We present here results from two simulations of mergers between two of these models. The first one is an equal mass merger. The second one is a merger with mass ratio 3:1. We place the systems on elliptical orbits to ensure merging. Both systems are placed on penetrating orbits with disks orientations: $\left(\theta_{1}, \phi_{1}\right),\left(\theta_{2}, \phi_{2}\right)=(70,30),(10,-10)$. The $z$-component of the spin angular momentum is then parallel to the orbital angular momentum. The spin-orbit coupling is thus large, although it is not maximum.

Each simulation consists of $10^{6}$ particles in total. We have run these simulations with the parallel version of GADGET (Springel et al. 2001) on a Beowulf cluster with 16 CPU's. Each run takes of the order of $5 \times 10^{5}$ seconds of CPU time. After merger is completed we let the remnants to evolve in isolation for 10 half-mass radius crossing times.

\section{Results}

We have obtained LOSVD's in the following way. We have binned the slit in ten spatial bins. We find the radial projected velocity and find the number of particles in each bin in velocity for each bin in the slit. In this way we find a line-of-sight velocity distribution. Finally we fit the LOSVD first by a Gaussian and the residuals by a Gauss-Hermite polynomial as given by Bender et al. (1994). We repeated this for 90 different points of view for each model.

Bender et al. (1994) find that for low values of $V / \sigma$ there is large scatter in values of H3. Most boxy ellipticals are located here. Disky ellipticals show large $V / \sigma$ with large values of $\mathrm{H} 3$.

Figure 1 shows that the inner parts of the 1:1 merger remnant do not show the correlation found by Bender et al., in agreement with Naab \& Burkert (2001). However, when inspecting at a larger radius, the general shape of Bender's correlation appears. This may indicate the presence of disk material in the outer parts of the remnant. 

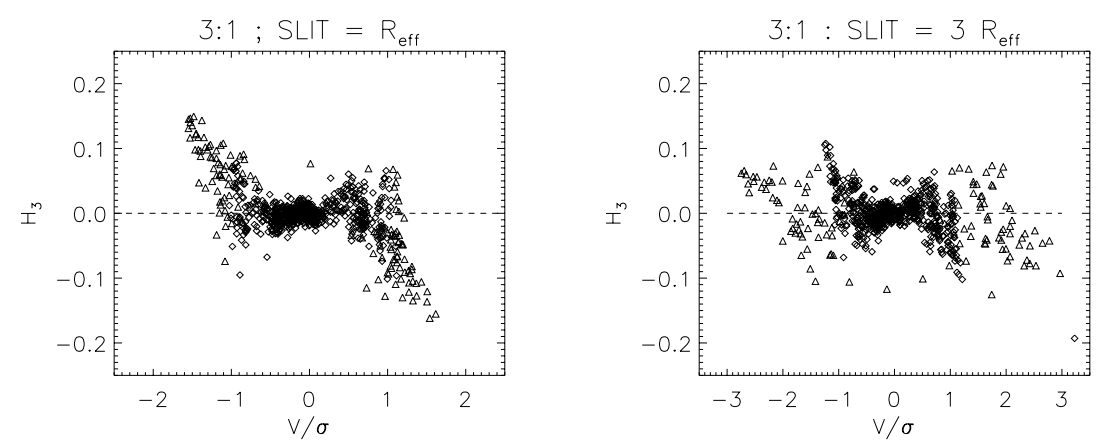

Figure 2. H3 vs. $V / \sigma$ relation for the $3: 1$ merger, again for a slit with length equal to $R_{e} f f$ (left) and for $3 R_{\text {eff }}$ (right).

Figure 2 left panel shows a similar trend to that found by Bender et al. The agreement is best for the outer parts (large $V / \sigma$ ). We fail to reproduce the large values observed by Bender et al. for low values of $V / \sigma$. The right panel gives a prediction of how the plot would be for slits including up to $3 R_{\text {eff }}$ (triangles).

\section{Discussion}

Collisionless equal mass mergers of disks are unable to reproduce the profile observed in real elliptical galaxies, in agreement with Naab \& Burkert (2001). 3:1 mergers on the other hand seem to follow the trend for large $V / \sigma$, in disagreement with Naab \& Burkert. Our initial models have a larger bulge. This helps to stabilize the disk during the merger (see Gonzalez-Garcia \& Balcells 2004).

We are not able to find large values of $\mathrm{H} 3$ for the inner parts. Our initial bulges do not rotate. We are carrying out new experiments with rotating bulges to test whether this may help to solve this discrepancy. In the outer parts of the 3:1 merger remnant we can find material from the disk of the large system. Thus, the H3 vs. $V / \sigma$ relation might be the signature in the LOSVD of the rotation of the initial large disk.

\section{Acknowledgements}

We would like to acknowledge Konrad Kuijken and John Dubinski for making their code available.

\section{References}

Bender R., Saglia R. P., Gerhard O. E. 1994 MNRAS 269, 785-813.

Bendo G.\& Barnes J. E. 2000 MNRAS 319, 315-325.

Gonzalez-Garcia A. C. \& Balcells M. 2004 in preparation.

Kuijken K. \& Dubinski J. 1995 MNRAS 277, 1341-1353.

Naab T. \& Burkert A. 2001 ApJ 555, L91-L94.

Springel V., Yoshida N., White S. D. M. 2001 New Ast. 6, 79-117.

Toomre A. \& Toomre J. 1972 ApJ 178, 623-666. 